\title{
EXTINCTION AND EXPLOSION OF NONLINEAR MARKOV BRANCHING PROCESSES
}

\author{
ANTHONY G. PAKES
}

(Received 28 April 2005; revised 12 April 2006)

Communicated by V. Stefanov

\begin{abstract}
This paper concerns a generalization of the Markov branching process that preserves the random walk jump chain, but admits arbitrary positive jump rates. Necessary and sufficient conditions are found for regularity, including a generalization of the Harris-Dynkin integral condition when the jump rates are reciprocals of a Hausdorff moment sequence. Behaviour of the expected time to extinction is found, and some asymptotic properties of the explosion time are given for the case where extinction cannot occur. Existence of a unique invariant measure is shown, and conditions found for unique solution of the Forward equations. The ergodicity of a resurrected version is investigated.
\end{abstract}

2000 Mathematics subject classification: primary 60J75, 60J80; secondary 60J25.

Keywords and phrases: Markov branching process, regular Markov process, population process, explosion, invariant measure and distribution.

\section{Introduction}

Let $\left\{p_{j}: j=0,1, \ldots\right\}$ be a probability mass function satisfying $p_{j}<1$ for all $j$ and $p_{1}=0$, and denote its probability generating function by $f(s)=\sum_{j \geq 0} p_{j} s^{j}$. Next, let $\lambda(j)>0$ for natural numbers $j$ and $\lambda(0)=0$. Our principal objective is to examine extinction and explosion of the minimal Markov process $\mathcal{X}:=\left(X_{t}: t \geq 0\right)$ with the non-negative integers as state space, and whose q-matrix $Q$ has elements

$$
q_{i j}= \begin{cases}\lambda(i) p_{j-i+1} & \text { if } i>0, j \geq 0 \text { and } i \neq j \\ -\lambda(i) & \text { if } i=j \geq 1 \\ 0 & \text { otherwise }\end{cases}
$$

(C) 2007 Australian Mathematical Society 1446-7887/07 \$A2.00+0.00 
Let $m=f^{\prime}(1-) \leq \infty$ and let $q$ be the least non-negative solution of $s=f(s)$. The jump chain determined by the q-matrix (1.1) is an unrestricted random walk $\mathcal{S}=\left\{S_{n}: n \geq 0\right\}$ that is skip-free to the left, sometimes called left-continuous. Let $N=\inf \left\{n: S_{n}=0\right\}$ and $\tau_{0}=\inf \left\{t: X_{t}=0\right\}$ be the zero-state hitting times of $\mathcal{S}$ and $\mathcal{X}$, respectively. Denoting $P_{i}(\cdot)=P\left(\cdot \mid X_{0}=i\right)$, the known fact $P_{i}(N<\infty)=q^{i}$ implies that $P_{i}\left(\tau_{0}<\infty\right)=q^{i}$, irrespective of the form of the jump rates $\lambda(i)$.

Let $\tau_{e}=\sup \left\{t>0: X_{t}<\infty\right\}$ be the explosion time of the minimal process. We say that $\mathcal{X}$ is regular if $P_{i}\left(\tau_{e}=\infty\right)=1$, and irregular otherwise. If $\mathcal{X}$ is irregular then the backward Kolmogorov (BK) sytem of equations has many solutions, and this may also be true for the forward Kolmogorov (FK) system. Examination of these possibilities is the main objective of this paper. To set the context we look first at what is presently known.

The minimal process $\mathcal{Z}:=\left(Z_{t}: t \geq 0\right)$ for the case $\lambda(i)=a i$, where $a>0$, is the Markov branching process (MBP), which allows the interpretation that $Z_{t}$ is the size at time $t$ of a population of individuals whose lifetimes have an exponential law with density $a e^{-a x}$ and which, at the end of their lives, produce $j$ offspring with probability $p_{j}$. All lifetimes and progeny numbers are independent. This physical model implies that family lineages develop independently of each other, the branching property. This is expressed analytically by the generating function identity

$$
F_{i}(s, t):=E_{i}\left(s^{Z_{t}}\right)=\left[F_{1}(s, t)\right]^{i}, \quad(i=0,1, \ldots),
$$

where $E_{i}(\cdot)$ denotes expectation conditional on the initial state $i$. The BK system has only one solution with the branching property, and this solution uniquely solves the FK system (Harris [15, page 97]). Hence only the minimal process bears the above interpretation as a model of population growth. It follows also that the zero state is absorbing, corresponding in the physical model to population extinction. We write $F(s, t)=F_{1}(s, t)$.

The BK system for the MBP can be expressed in integrated form as

$$
\int_{s}^{F(s, t)} \frac{d u}{f(u)-u}=a t, \quad(0 \leq s \leq 1),
$$

where the integral is defined by continuity if $s=q$. Since $P_{i}\left(Z_{i}<\infty\right) \equiv 1$ if and only if $P_{i}\left(\tau_{e}=\infty\right)=1$, we see that by letting $s \uparrow 1$ that the MBP is regular if and only if for each $\epsilon \in(0,1-q)$, the integral

$$
I=\int_{1-\epsilon}^{1} \frac{d s}{s-f(s)}
$$

is divergent. This criterion usually is named after Harris [15] who gave this analytical proof in more detail. Harris mentioned that the criterion appeared without proof in 
Zolotarev [31, Equation (1)], and also that it was attributed there to Dynkin. Harris' reference was to the Russion version, and the Dynkin attribution was not included in the English translation. Savits [29] called this regularity condition 'a well known result of Dynkin', but his only reference was to Harris' attribution. In addition, Savits mentioned a probabilistic proof in unpublished Stanford lecture notes of Ikeda. This proof probably is the same as that in Ikeda et al. [16, page 395]. Properties of the irregular MBP have been examined by Pakes [25] and Sagitov [28].

We seek general criteria for regularity of the nonlinear MBP, defined as the minimal process corresponding to (1.1). The most far-reaching generalization of the MBP admits any q-matrix subject to the skip-free property $q_{i j}=0$ if $j<i-1$ (and which is inherent in (1.1)). Lenz [19] considered this most general model and he obtained a condition for regularity under the additional constraint that there is a constant $K \geq 1$ such that $q_{i j}=0$ if $j-i>K \geq 1$, that is, upward jumps are bounded. He also considered the particular case of (1.1) with

$$
\lambda(i)=a i^{\theta},
$$

where $1 / 2<\theta \leq 1$ is a constant and, it seems, $p_{j-i+1}=0$ if $j-i>K$. This, case is always regular. In fact, even without restricting jump sizes, and assuming only $0<\theta \leq 1$, the minimal process in this case is stochastically smaller than the MBP (the case $\theta=1$ ), and hence $\left(X_{t}\right)$ is regular if the MBP is.

Kuster [18] allowed a completely general jump chain, but restricted the jump rate to be linear, as for the MBP, or sublinear in the sense that there exists $\theta \in[0,1]$ such that $\left\{i^{-\theta} \lambda(i): i \geq 1\right\}$ is non-increasing and $\lambda(i) / i \rightarrow 0$. The regularity problem here is open, although the moment conditions imposed by Kuster [18] ensure his process is regular.

Reinhard [27] retained the skip-free property of the jump chain used by Lenz [19] and she assumeed (1.4) with $0<\theta \leq 1$. However once again her moment conditions ensure regularity, and her interest centered on diffusion approximations under the near-criticality condition $\sum_{j \neq i} j q_{i j} / \lambda(i)=1+O\left(i^{-1+\beta}\right)$ for some $\beta>0$. This work seems to be independent of Lipow [20].

The motivation for the above generalizations of the MBP is that in some (undefined?) sense they capture the growth in numbers of individuals whose lifetime and/or splitting laws depend on instantaneous population size. A mathematical motivation is finding perturbations of the MBP whose properties are qualitatively similar to the MBP.

Quite independently of all this earlier work, Chen [9] considered the model (1.1), including the case (1.4) with $0<\theta \leq 1$. His motivation came partly from controlled branching processes and more particularly from (1.1) with $\lambda(i)=i^{2}$, a process said to be associated with the dual of the Fleming-Viot process (Etheridge [12]). Chen [9] proved regularity under various conditions. In particular, he showed that if (1.4) holds 
and $1<m<\infty$, then $\mathcal{X}$ is regular if and only if $0<\theta \leq 1$. This assertion follows also from the general criteria for regularity of jump Markov processes proved using martingale arguments by Kersting and Klebaner [17], and Hamza and Klebaner [14] should be mentioned in this connection.

Chen [5] and Chen et al. [6] examined some properties of the generalized MBP, defined as the minimal process corresponding to (1.1) and (1.4) with $\theta>0$. The latter reference established an integral test, which subsumes the Harris-Dynkin test. They used an analytical approach based on identities satisfied by transition probabilities and resolvent elements.

In this paper we provide a more probabilistic approach, which substantially reduces the above assumptions about the jump rates. The case $m \leq 1$ is seen in Section 2 to almost surely (a.s.) hit $\{0\}$, and hence be regular. We obtain a substantially complete description of the asymptotic behaviour of the expected hitting time. In Section 3 we obtain necessary and sufficient conditions for regularity assuming only that $\{\lambda(j): j \geq 1\}$ is ultimately non-decreasing. In particular, a generalization of $(1.4)$ in which $\{1 / \lambda(j)\}$ is a moment sequence gives a rather natural extension of the Harris-Dynkin criterion. For the irregular case we give some simple results on asymptotics for the explosion time which show the variety of behaviours that are possible. After completing this research Dr A. Chen sent me a copy of Chen et al. [7], which contains results overlapping those in Sections 3 and 5 below, but with substantial differences in their proofs. In particular, these authors developed a test sequence for assessing whether or not the FK system is uniquely satisfied by the minimal transition semigroup. This test was used by Chen et al. [8] to derive a simple necessary and sufficient condition for such uniqueness. In Section 5 we provide what we believe is a more direct proof.

Existence and form of stationary measures for the transition semigroup of $\mathcal{X}$ is addressed in Section 4 . Resurrected forms of $\mathcal{X}$ are constructed by allowing $q_{0 j}>0$ for at least one value of $j>0$. Chen [4] considers regularity and ergodicity properties when (1.4) holds, and in Section 6 we extend these results to arbitrary jump rates.

\section{The case $m \leq 1$}

If $m \leq 1$, then $p_{0}>0$ and $q=1$ and hence $S$ will hit the zero state, which is absorbing for $\mathcal{X}$ under the sole condition that $\lambda(0)=0$. The following result is immediate. Recall that $\tau_{0}$ is the hitting time of $\{0\}$. and let $q_{i}=P_{i}\left(\tau_{0}<\infty\right)$.

THEOREM 2.1. Suppose (1.1) holds with $\lambda(0)=0$ and $\lambda(i)>0$ for all positive $i$. If $m \leq 1$, then the minimal process $\mathcal{X}$ is regular and $q_{i} \equiv 1$. 
Let $\left\{A_{n}: n \geq 0\right\}$ denote the random walk $\mathcal{S}$ stopped when it first hits $\{0\}$,

$$
A_{n}= \begin{cases}S_{n} & \text { if } S_{0}, \ldots, S_{n}>0 \\ 0 & \text { otherwise }\end{cases}
$$

Denote its transition probabilities by $a_{i j}^{(n)}=P_{i}\left(A_{n}=j\right)$, and the mean occupation time of state $\{j\}$ by $A_{i j}=\sum_{n \geq 0} a_{i j}^{(n)}$, which is finite if $j \geq 1$. It is clear that

$$
H_{i}:=E_{i}\left(\tau_{0}\right)=\sum_{j \geq 1} A_{i j} / \lambda(j)
$$

Discussion of properties of $H_{i}$ requires the following facts about the stopped random walk; see Pakes [21] and references cited there.

First, $E_{i}\left(x^{N}\right)=g^{i}(x)$ where $g$ is the unique solution of the functional equation $g=x f(g)$. Let $\alpha_{i}^{(n)}(s)=E_{i}\left(s^{A_{n}}\right)$. Pakes [21, Section 3] showed that

$$
\alpha_{i}^{(n+1)}(s)=b(s) \alpha_{i}^{(n)}(s)-(b(s)-1) a_{i 0}^{(n)},
$$

where $b(s)=f(s) / s$. It follows that

$$
A_{i}(s, x):=\sum_{n=0}^{\infty} \alpha_{i}^{(n)}(s) x^{n}=\frac{s^{i}-(b(s)-1) x A_{i}(0, x)}{1-x b(s)}
$$

and since the denominator is zero when $s=g(x)$ we obtain

$$
A_{i}(0, x)=\sum_{n=0}^{\infty} a_{i 0}^{(n)} x^{n}=\frac{g^{i}(x)}{1-x}
$$

Subtraction yields

$$
\sum_{n=0}^{\infty} E_{i}\left(s^{A_{n}} ; A_{n}>0\right) x^{n}=A_{i}(s, x)-A_{i}(0, x)=\frac{s^{i}-g^{i}(x)}{1-x b(s)},
$$

and since $g(1-)=1$ if $m \leq 1$, we obtain the following generating function for $A_{i j}$,

$$
\sum_{j \geq 1} A_{i j} s^{j}=s \frac{1-s^{i}}{f(s)-s}
$$

The coefficients can be written explicitly as

$$
A_{i j}=u_{j-i}+\cdots+u_{j-1}
$$

where $\left\{u_{j}\right\}$ is the renewal sequence with the generating function $(1-w(s))^{-1}$ and $w(s)=(1-f(s)) /(1-s)$. The associated renewal process is transient if $m<1$ and recurrent if $m=1$. In (2.5) we define $u_{j}=0$ if $j<0$. 
This representation allows us to determine the asymptotic behaviour of $H_{i}$, and hence understand the rôle of the jump rates in this behaviour. It is worth recalling for the MBP case that

$$
H_{i} \sim \frac{\log i}{a(1-m)}, \quad(i \rightarrow \infty),
$$

if $m<1$, and this can be refined under further moment conditions; see (2.6) in Pakes [23]. If $m=1$ and $f^{\prime \prime}(1)<\infty$, then $H_{i} \equiv \infty$. We deal separately with the subcritical $(m<1)$ and critical $(m=1)$ cases.

THEOREM 2.2. Suppose $m<1$, and let $\Lambda(i)=\sum_{j=1}^{i} 1 / \lambda(j), \Lambda(0)=0$, and $\Lambda=\lim _{i \rightarrow \infty} \Lambda(i)$.

(a) If $\Lambda<\infty$, then $\lim _{i \rightarrow \infty} H_{i}=\Lambda /(1-m)-\sum_{j \geq 1} u_{j} \Lambda(j)$.

(b) Suppose that $\Lambda=\infty, \beta:=\inf _{j \geq 1} \lambda(j)>0$, and that there is a positive constant $M$ such that

$$
\sup _{l \geq 1} \frac{\Lambda(i+l)-\Lambda(i)}{\Lambda(l)} \leq M
$$

uniformly for $i \geq 1$. Then $H_{i}<\infty$ for all $i$ and $H_{i} \sim \Lambda(i) /(1-m)$.

PROOF. Observe that (2.1) and (2.5) can be expressed as

$$
H_{i}=D_{1 i}+D_{2 i}:=\sum_{j=1}^{i} \frac{1}{\lambda(j)} \sum_{l=0}^{j-1} u_{l}+\sum_{j>i} \frac{1}{\lambda(j)} \sum_{l=j-i}^{j-1} u_{l}
$$

Using the fact that $\sum_{j \geq 0} u_{j}=(1-m)^{-1}$, we see for case (a) that

$$
D_{1 i} \rightarrow \sum_{j \geq 1} \frac{1}{\lambda(j)} \sum_{l=0}^{j-1} u_{l}=\sum_{l \geq 0} u_{l}(\Lambda-\Lambda(l)),
$$

which equals the asserted limit form. Similarly, $D_{2 i}$ is dominated by

$$
\frac{1}{1-m} \sum_{j>i} \frac{1}{\lambda(j)} \rightarrow 0
$$

For case (b) write

$$
D_{1 i}=\frac{\Lambda(i)}{1-m}-\sum_{j=1}^{i} \frac{1}{\lambda(j)} \sum_{l \geq j} u_{l} .
$$

For a given $\epsilon>0$ choose $i^{\prime}$ so that $\sum_{l>i^{\prime}} u_{l}<\epsilon$, and observe that the double sum $D_{3 i}$ is dominated by $(1-m)^{-1} \sum_{j=1}^{i^{\prime}} 1 / \lambda(j)+\epsilon \Lambda(i)$. Hence $D_{3 i}=o(\Lambda(i))$. Reversing 
the order of summation for $D_{2 i}$ yields $D_{2 i}=\Delta_{1 i}+\Delta_{2 i}$ where

$$
\Delta_{1 i}=\sum_{l=1}^{i} u_{l} \sum_{j=i+1}^{i+l} \lambda^{-1}(j)=\sum_{l=1}^{i} u_{l}(\Lambda(i+l)-\Lambda(i))
$$

and

$$
\Delta_{2 i}=\sum_{l>i} u_{l}(\Lambda(i+l)-\Lambda(l))
$$

It follows from (2.6) that $\Delta_{1 i} \leq M \sum_{l=1}^{i} u_{l} \Lambda(l) \leq M\left[\sum_{l=1}^{i^{\prime}} u_{l} \Lambda(l)+\epsilon \Lambda(i)\right]$, so $\Delta_{1 i}=o(\Lambda(i))$. Observe that $\Lambda(i+l)-\Lambda(l) \leq i / \beta$ and hence $\Delta_{2 i}<\infty$ for all $i$. Interchanging the rôles of $i$ and $l$ in (2.6) yields the bound

$$
\Delta_{2 i} \leq M \Lambda(i) \sum_{l>i} u_{l}=o(\Lambda(i))
$$

thus completing the proof.

Observe that $\Delta_{2 i}>\sum_{l>i} u_{l} / \lambda(l+1)$ and that if $\beta=0$, then it is possible that the series diverges, that is, that $H_{i} \equiv \infty$. If the series converges, and if $\{\lambda(j)\}$ is ultimately non-decreasing, then $H_{i}<\infty$. In addition, (2.6) holds if $\{\lambda(j)\}$ is ultimately non-decreasing.

THEOREM 2.3. Suppose $m=1$ and $\gamma=2 / f^{\prime \prime}(1)>0$. Then $H_{i} \equiv \infty$ if $\Lambda=\infty$, and $H_{i}<\infty$ for all $i$ if $\Lambda<\infty$. In the latter case:

(a) If $\sum_{j \geq 1} j / \lambda(j)<\infty$, then $\lim _{i \rightarrow \infty} H_{i}=\sum_{j \geq 1}(1 / \lambda(j)) \sum_{l=0}^{j-1} u_{l}<\infty$;

(b) If $\sum_{j \geq 1} j / \lambda(j)=\infty$, then

$$
H_{i} \sim \gamma[\mathcal{L}(i)+i(\Lambda-\Lambda(i))]
$$

where $\mathcal{L}(i)=\sum_{j=1}^{i} j / \lambda(j)$.

Proof. Since $w^{\prime}(1)=\gamma^{-1}$, the discrete renewal theorem asserts that $u_{i} \rightarrow \gamma$. Observe that

$$
D_{2 i}=\gamma i(\Lambda-\Lambda(i))+\sum_{j>i} \frac{1}{\lambda(j)} \sum_{l=j-i}^{j-1}\left(u_{l}-\gamma\right) .
$$

There are $i$ summands in the inner sum, and they are smaller in modulus than $\epsilon$ if $l>l(\epsilon)$. It follows that the double sum equals $o(i(\Lambda-\Lambda(i))+O(\Lambda-\Lambda(i))$. Thus $D_{2 i} \sim \gamma i(\Lambda-\Lambda(i))$ for both cases (a) and (b).

In case (a) it is clear that $i(\Lambda-\Lambda(i)) \leq \sum_{j \geq i} j / \lambda(j) \rightarrow 0$, implying $D_{2 i} \rightarrow 0$, and that $\lim _{i \rightarrow \infty} D_{1 i}<\infty$ since its inner sum $\sim j \gamma$ as $j \rightarrow \infty$. In case (b) $D_{1 i} \sim \gamma \mathcal{L}(i)$, and the assertion follows. 
EXAMPLE 2.4. Suppose $m=1, \gamma>0$, and that $\lambda(i)=i^{\theta} L(i)$ where $L(x)$ is slowly varying at infinity, and either $\theta>1$, or $\theta=1$ and $\Lambda<\infty$. We have case (a) of Theorem 2.3 if $\theta>2$, or if $\theta=2$ and $\sum_{i} 1 / i L(i)<\infty$, and we have case (b) otherwise. In case (b) we have $i(\Lambda-\Lambda(i)) \sim i^{2-\theta} /(\theta-1) L(i)$. If $1 \leq \theta<2$, then $\mathcal{L}(i) \sim i^{2-\theta} /(2-\theta) L(i)$, and hence

$$
H_{i} \sim \frac{\gamma i^{2-\theta}}{(2-\theta)(\theta-1) L(i)} .
$$

If $\theta=2$, then $\mathcal{L}(i)=\sum_{j=1}^{i} 1 / j L(j)$ diverges to infinity in a slowly varying manner. If $0<\epsilon<1$, then

$$
L(i) \mathcal{L}(i) \geq \sum_{\epsilon i \leq j \leq i} L(i) / j L(j) \sim \sum_{\epsilon i \leq j \leq i} 1 / j \rightarrow \log (1 / \epsilon),
$$

where we have used the uniform convergence theorem for slowly varying functions. Letting $\epsilon \rightarrow 0$, we conclude that $H_{i} \sim \gamma \mathcal{L}(i)$. In particular, if $L(j) \rightarrow a$, then $H_{i} \sim(\gamma / a) \log i$.

An explicit integral representation for $H_{i}$ follows by assuming there exists a measure $\mu$ supported in the unit interval $[0,1]$ satisfying (i) $\mu((0,1))>0$, and (ii):

$$
\lambda(i)=\left[\int_{0}^{1} s^{i-1} \mu(d s)\right]^{-1} .
$$

Assumption (i) ensures that $\mu$ is not concentrated at the origin. The jump rates are unboundedly increasing if and only if $\mu(\{1\})=0$. Integrating (2.4) with respect to $\mu$ yields the following result.

THEOREM 2.5. Suppose (1.1) and (2.8) hold and $m \leq 1$. Then the expected time to extinction is

$$
H_{i}=\int_{0}^{1} \frac{1-s^{i}}{f(s)-s} \mu(d s) .
$$

Taking $\mu(d s)=a^{-1} d s$ in (2.8) gives the MBP case $\lambda(i)=a i$; see Pakes [22, Equation (3.3)]. If $\theta>0$ and

$$
\mu(d s)=\frac{(\log (1 / s))^{H-1}}{a \Gamma(\theta)} d s,
$$

then (1.4) holds; see Chen [5, Equation (4.2)]. The asymptotic forms in Example 2.4 could be obtained from (2.9) by assuming that $\mu(d s)=\zeta(s) d s$, where $\zeta(x)$ is regularly varying at zero. It is obvious that

$$
\lim _{i \rightarrow \infty} H_{i}=\int_{0}^{1} \frac{\mu(d s)}{f(s)-s} \leq \infty .
$$




\section{The super-critical case, $m>1$}

In this section we assume $1<m \leq \infty$, in which case the jump chain $\left\{A_{n}\right\}$ drifts to $\infty$ with probability $1-q^{i}$ if $X_{0}=i$, and then explosion may occur if the jump rates grow sufficiently fast. If this occurs for the MBP, then the BK system has many distinct solutions, although it is known that the FK system is satisfied only by the minimal solution (Harris (1963)). Let $\mathcal{I}=\{N=\infty\}=\{X, \rightarrow \infty\}$. A small adaptation of the well-known explosion criterion for jump Markov processes asserts that $\phi_{i}:=P_{i}\left(\tau_{e}<\infty \mid \mathcal{I}\right)=1$ if and only if

$$
P_{i}\left(\sum_{n \geq 1} \frac{1}{\lambda\left(A_{n}\right)}<\infty \mid \mathcal{I}\right)=1 .
$$

We can apply this criterion immediately to dispose of one case, since the series diverges a.s. on $\mathcal{I}$ if the jump rates are bounded.

LEMMA 3.1. If $1<m \leq \infty$ and $\{\lambda(j)\}$ is bounded, then $\mathcal{X}$ is regular.

In general, what we may call a Feller-Lundberg type of criterion would assert that (3.1) holds if and only if

$$
\eta_{i}:=E_{i}\left(\tau_{e} \mid \mathcal{I}\right)=\sum_{n \geq 1} E_{i}\left[1 / \lambda\left(A_{n}\right) \mid \mathcal{I}\right]<\infty
$$

Obviously (3.2) always implies (3.1), and the burden of the following proofs is to give conditions under which the converse holds. See Pakes [24, Section 2] for an elementary example of a random series of independent summands that converges, but has infinite mean.

We begin by investigating conditions for (3.2). Observe that $P_{i}\left(A_{n}=j \mid \mathcal{I}\right)=$ $a_{i j}^{(n)}\left(1-q^{j}\right) /\left(1-q^{i}\right)$, and hence that

$$
\eta_{i}=\sum_{j \geq 1}(\lambda(j))^{-1} A_{i j} \frac{1-q^{j}}{1-q^{i}} .
$$

Observing that $g(1-)=q$ if $m>1$, we have the following replacement for (2.4),

$$
\sum_{j \geq 1} A_{i j} s^{j}=s \frac{q^{i}-s^{i}}{f(s)-s},
$$

which yields the following result. 
THEOREM 3.2. If $1<m \leq \infty$ and (2.8) both hold, then

$$
\eta_{i}=\left(1-q^{i}\right)^{-1} \int_{0}^{1} s^{-1}\left[\frac{s^{i}-q^{i}}{1-b(s)}-q^{i} \frac{1-s^{i}}{1-b(q s)}\right] \mu(d s) .
$$

In particular, $\eta_{i}=\infty$ for all $i \geq 1$ if and only if

$$
J:=\int_{1-\epsilon}^{1} \frac{\mu(d s)}{s-f(s)}=\infty
$$

for any $\epsilon \in(0,1-q)$.

Theorem 3.2 implies that $\mathcal{X}$ is irregular if (2.8) holds and $J<\infty$. The next result proves the converse, thus giving a direct generalization of the Harris-Dynkin integral criterion, as well as generalizing the integral criterion obtained with analytic arguments based on the resolvent by Chen et al. [6]. We use the following result.

LEMMA D. Let $p_{0}=0, S_{0}=0$ and $S_{n}=V_{1}+\cdots+V_{n}$ where $\left\{V_{n}: n \geq 1\right\}$ is an independent sequence of $\mathbb{N}$-valued random variables having the probability generating function $f(s) / s$. If $0<\lambda(j) \rightarrow \infty$ as $j \rightarrow \infty$ and there exists $j^{\prime}$ such that $\lambda(j+1) \geq \lambda(j)\left(j \geq j^{\prime}\right)$, then for each $i \in \mathbb{N}$,

$$
\sum_{n \geq 1} 1 / \lambda\left(i+S_{n}\right)=\infty \text { if and only if } \sum_{n \geq 1} E\left[1 / \lambda\left(i+S_{n}\right)\right]=\infty .
$$

This is essentially part (ii) of the theorem in Doney [10]. He assumed that $\{\lambda(j)\}$ is non-decreasing, but in our case altering details of his proof shows that this is not necessary, nor is his condition (*) required. We will say that the rate sequence is ultimately monotone (and hence non-decreasing) if it satisfies the conditions in Lemma D.

THEOREM 3.3. Suppose that (2.8) holds for the q-matrix (1.1), that $\mu(\{1\})=0$, and that $1<m \leq \infty$. Then the minimal process is regular if and only if (3.6) holds.

Proof. We consider two cases. (i) If $p_{0}=0$ then $\left\{i+S_{n}\right\}$ is the jump chain and the assertion follows directly from Lemma $\mathrm{D},(3.1)$ and Theorem 3.2.

(ii) Suppose $0<p_{0}<1$. We prove that $J=\infty$ implies that $\mathcal{X}$ is regular by stochastic comparison with the case $p_{0}=0$. Let $r_{0}=1-p_{0}, \hat{f}(s)=\left(f(s)-p_{0}\right) / r_{0}$, $\hat{q}_{i j}=q_{i j} / r_{0}$ if $j>i, \hat{q}_{i j}=0$ if $j<i$, and $\hat{q}_{i i}=-q_{i i}$. The minimal process corresponding to the q-matrix $\hat{Q}$ is a generalized MBP and, from case (i), it is regular if and only if

$$
\int_{0}^{1} \frac{\mu(d s)}{s-\hat{f}(s)}=r_{0} \int_{0}^{1} \frac{\mu(d s)}{s-f(s)+p_{0}(1-s)}=\infty .
$$


The denominator in the second integral is asymptotically proportional to $s-f(s)$ as $s \rightarrow 1-$, so it follows from (3.6) that $\hat{\mathcal{X}}$ is regular if and only if (3.6) holds.

It is easily checked that $\sum_{j \geq k} \hat{q}_{i j} \geq \sum_{j \geq k} q_{i j}$ for all $i, k \geq 0$ and hence

$$
\sum_{k \leq j} \hat{p}_{i j}(t) \leq \sum_{k \leq j} p_{i j}(t)
$$

This assertion follows from the proof of Kirstein's theorem as presented by Anderson [1, page 250]. Specifically, the inequality holds for the approximating transition probabilities formed from $n \times n$ north-west truncations of $Q$ and $\hat{Q}$, and the assertion follows by letting $n \rightarrow \infty$ and using [1, Proposition 2.2.14]. Now let $k \rightarrow \infty$ to obtain the inequality

$$
P_{i}\left(\hat{X}_{t}<\infty\right) \leq P_{i}\left(X_{t}<\infty\right)
$$

and hence regularity of $\mathcal{X}$ is implied by that of $\hat{\mathcal{X}}$, in other words, (3.6) implies that $\mathcal{X}$ is regular.

EXAMPLE 3.4. If $1<m<\infty$, then (3.6) holds if and only if $\int_{0}^{1} \mu(d s) /(1-s)=\infty$. It follows from (2.8) that $\mathcal{X}$ is regular if and only if

$$
\sum_{j \geq 1} \frac{1}{\lambda(j)}=\infty .
$$

EXAMPLE 3.5. Suppose $\theta>0$ and (2.10) holds. Since $\log (1 / s) \sim 1-s$ as $s \rightarrow 1$, $\mathcal{X}$ is regular if and only if

$$
J_{\theta}:=\int_{1-\epsilon}^{1} \frac{(1-s)^{\theta-1}}{s-f(s)} d s=\infty .
$$

This criterion is derived by different means in Chen et al. [6, see Equations (2.3) and (2.4)]. It follows that $\mathcal{X}$ is irregular if $\theta>1$, and it is regular if $\theta<1$ and $m<\infty$. Suppose that $m=\infty$ and $1-f(s)=(1-s)^{\alpha} L\left((1-s)^{-1}\right)$ where $0 \leq \alpha \leq 1$ and $L$ is slowly varying at infinity. Then $s-f(s) \sim 1-f(s)$ and hence $J_{\theta}=\infty$ if and only if

$$
\tilde{J}_{\theta}=\int_{1 / \epsilon}^{\infty}\left(x^{1+\theta-\alpha} L(x)\right)^{-1} d x=\infty .
$$

Hence $\mathcal{X}$ is regular if $\theta<\alpha$ and irregular if $\theta>\alpha$. Either outcome can occur if $\theta=\alpha$. These conclusions remain intact if $\mu(d s)=(1-s)^{\theta-1} M\left((1-s)^{-1}\right)$, where $M(x)$ is slowly varying at infinity, provided $\theta \neq \alpha$. If $\theta=\alpha$, then $\mathcal{X}$ is regular if and only if $\int_{1}^{\infty}(M(x) / x L(x)) d x=\infty$. 
The outcome we found in Example 3.4 holds without any structural assumption on the jump rates.

THEOREM 3.6. If $1<m<\infty$, then $\mathcal{X}$ is regular if and only if (3.8) holds.

In order to explain this, and for other purposes, we need the following notation and facts. The function $w(s ; q)=(q-f(s)) /(q-s)$ is a probability generating function. To see this observe that $w(1)=1$ and, if $q>0$, then $w(q s ; q)=$ $(1-f(q s) / q) /(1-s)$ is the generating function of the terms $\sum_{l>j} p_{l} q^{l-1} \geq 0$. Write $w(s ; q)=\sum_{j \geq 1} w_{j}(q) s^{j}$ and observe that

$$
w_{j}(q)=q^{-j} \sum_{l>j} p_{l} q^{l-1}
$$

$w^{\prime}(1 ; q)=(m-1) /(1-q)$, and that $w_{0}(q)=1-p_{0} / q$ (where we adopt the convention that $p_{0} / q=1$ if $\left.p_{0}=0\right)$. Thus $(1-w(s ; q))^{-1}=\sum_{j \geq 0} u_{j}(q) s^{j}$ where $\left\{u_{j}(q)\right\}$ is a (generalized) renewal sequence, and hence the discrete renewal theorem yields $u_{\infty}(q):=\lim _{j \rightarrow \infty} u_{j}(q)=1 / w^{\prime}(1, q)$.

Theorem 3.6 is proved in the case $p_{0}>0$ by Chen et al. [7] without reference to (3.1) or to Reuter's (equivalent) analytical condition (Anderson [1, page 81]). They prove the following identity for the resolvent elements $r_{i j}(\theta)=\int_{0}^{\infty} p_{i j}(t) e^{-\theta t} d t$, valid if $p_{0} \geq 0$,

$$
u_{j-i}(q)-\theta \sum_{k=0}^{j-1} r_{i k}(\theta) u_{j-k}(q)=r_{i, j-1}(\theta) \lambda(j-1)-q r_{i j}(\theta) \lambda(j),
$$

which is obtained from the generating function form of the FK-system. Letting $j \rightarrow \infty$ it follows from the dominated convergence theorem that

$$
\lim _{j \rightarrow \infty}\left[r_{i, j-1}(\theta) \lambda(j-1)-q r_{i j}(\theta) \lambda(j)\right]=u_{\infty}(q) \Delta(\theta)
$$

where $\Delta(\theta)=1-\theta \sum_{k=0}^{\infty} r_{i k}(\theta)$. In particular, if $\mathcal{X}$ is irregular, then $\Delta(\theta)>0$, so choosing $0<\delta<\Delta(\theta)$ we find in the case $q=0$ that $1 / \lambda(j) \leq r_{i j}(\theta) / \delta u_{\infty}(0)$ if $j$ is sufficiently large and hence $\sum_{j} 1 / \lambda(j)<\infty$. On the other hand, it follows from (3.10) that there is a constant $C>u_{\infty}(q)>0$, independent of $\theta$ and $j$ such that if $j \geq 1$, then $r_{i j}(\theta) \leq C / \lambda(j)$. Summing over $j$ and assuming $\mathcal{X}$ is regular yields $\theta^{-1} \leq \sum_{j} 1 / \lambda(j)$. Letting $\theta \rightarrow 0$ shows that (3.8) holds. This argument completes the proof of Theorem 3.6 for the case $p_{0}=0$, and it follows the strategy used by Chen et al. [7].

In the results which follow, we show, subject to some mild technical conditions, that a necessary and sufficient condition ((3.11) below) for regularity is possible in the case $m=\infty$. The first such result places no condition on the jump rates, but it assumes a little about the offspring distribution. 
THEOREM 3.7. Suppose that $1<m \leq \infty$ and that one of the following conditions holds:

(a) $p_{0}>0$; or

(b) $p_{0}=0$ and $\lim \sup _{j \rightarrow \infty} p_{j+1} / p_{j}<\infty$.

Then either $\eta_{i}=\infty$ for all $i$ or $\eta_{i}<\infty$ for all $i$. The former occurs if and only if

$$
K(q):=\sum_{j \geq 0} \frac{u_{j}(q)}{\lambda(j+1)}=\infty .
$$

In particular, $\mathcal{X}$ is irregular if $K(q)<\infty$.

Proof. We see from (3.3) that $\eta_{i}=\infty$ if and only if $\sum_{j \geq 1} A_{i j} / \lambda(j)=\infty$. By expressing $f(s)$ in terms of $w(s ; q)$ in the right-hand side of $(3.4)$ it follows that

$$
A_{i j}=\sum_{k=1}^{i} q^{i-k} u_{j-k}(q) .
$$

If (a) holds, then $A_{i j} \geq q^{i-1} u_{j-1}(q)$ and hence $\eta_{i}<\infty$ implies that $K(q)<\infty$ for all $i$. Conversely, it is clear that if $K(q)<\infty$ then $\eta_{i}<\infty$ provided $L:=$ $\lim \sup _{j \rightarrow \infty} u_{j-1}(q) / u_{j}(q)<\infty$. The recursive relation $w_{j}(q)=q w_{j+1}(q)+p_{j+1}$ follows from (3.9), and it implies that $w_{j+1}(q) / w_{j}(q) \leq q^{-1}$. It follows from ratio estimates for renewal sequences (Theorem 2.3 of Garsia et al. [13]) that $M:=$ $\lim \sup _{j \rightarrow \infty} u_{j}(q) / u_{j-1}(q) \leq q^{-1}$, and hence that $L \leq(m q)^{-1}<\infty$, as desired. In the case of Assumption (b) the assertion follows because this additional hypothesis ensures that the quotients $u_{j-1}(0) / u_{j}(0)$ are bounded away from zero and infinity. The proof is complete.

Our next result pushes the analysis of Chen et al. [7] as far as possible, although the final outcome is unsatisfactory in the case $p_{0}>0$ because the regularity assumption used to make the proof work cannot easily be expressed in terms of the offspring distribution.

THEOREM 3.8. If $m=\infty$ and $\left\{w_{j}(q)\right\}$ is a log-convex sequence, then $\mathcal{X}$ is regular if and only if (3.11) holds

PROOF. de Bruijn and Erdös [3] show that the log-convexity condition implies the same property of the renewal sequence $\left\{u_{j}(q)\right\}$, and in particular that, for each $k$, $u_{j-k}(q) / u_{j}(q) \downarrow 1$ as $j \uparrow \infty$. Hence details of the last proof can be altered to conclude that $K(q)<\infty$ implies that $\eta_{i}<\infty$ for all $i \geq 1$, and conversely that $\eta_{i}<\infty$ for some $i$ implies that $K(q)<\infty$. In particular, $\mathcal{X}$ is irregular if $K(q)<\infty$. 
It follows from the above ratio limit conclusion and (3.10) that

$$
\lim _{j \rightarrow \infty} \frac{\left[r_{i, j-1}(\theta) \lambda(j-1)-q r_{i j}(\theta) \lambda(j)\right]}{u_{j}(q)}=\Delta(\theta)
$$

from which, assuming $\mathcal{X}$ is irregular, we obtain

$$
r_{i, j-1}(\theta) \lambda(j-1)-q r_{i j}(\theta) \lambda(j) \geq \delta u_{j}(q)
$$

if $j \geq j^{\prime}>0$, where $0<\delta<\Delta(\theta)$. If $p_{0}>0$, then multiplying by $q^{j-1}$ and summing yields

$$
r_{i j}(\theta) \lambda(j) \geq \delta \sum_{l \geq 0} u_{l+j+1}(q) q^{l} \sim \frac{\delta u_{j-1}(q)}{(1-q)}, \quad(j \rightarrow \infty),
$$

where we have used the monotone convergence theorem to obtain the last estimate. This conclusion remains valid in the case $p_{0}=0$. Hence we have the bound

$$
u_{j}(q) / \lambda(j+1)=O\left(r_{i, j+1}(\theta)\right)
$$

for all sufficiently large $j$, and it follows that $K(q)<\infty$. The proof is complete.

Theorem 3.6 implies that if $1<m<\infty$, then Lemma 3.1 holds if the boundedness condition there is replaced with $\liminf _{j \rightarrow \infty} \lambda(j)<\infty$. This is not the case if $m=\infty$. To see this, suppose $p_{0}>0$ and $K(q)<\infty$, in which case $\mathcal{X}$ is irregular. Since $u_{j}(q) \rightarrow 0$, there is an infinite subset $S$ of $\mathbb{N}$ such that $\sum_{j \in S} u_{j}(q)<\infty$, so we can choose $\lambda(j) \equiv \lambda>0$ for $j \in S$ and preserve the irregularity. It follows that $\mathbb{N}$ cannot be denumerably atomic (as defined by Breiman [2]) for the random walk jump chain if $m=\infty$. It is conceivable in the case $1<m<\infty$ that $\mathbb{N}$ is denumerably atomic for the jump chain because the expected occupation time of any infinite subset $S$ is infinite.

The proofs of Theorems 3.6-3.8 use, in one form or another, ratio limit theorems for discrete renewal sequences. It seems that such arguments cannot demonstrate equivalence of regularity and (3.11) simply because it is possible that the quotient $u_{j+1}(q) / u_{j}(q)$ oscillates unboundedly. In the sequel we let $U_{j}(q)=\sum_{i=1}^{j} u_{i}(q)$.

Doney [10] proved that the MBP is regular if and only if $\sum_{j \geq 1}\left(j M_{j}\right)^{-1}=\infty$ where $M_{j}=\sum_{k=0}^{j} \sum_{l>k} p_{l}$. For the case $p_{0}=0$ (in which case $q=0$ ) this follows from the Harris-Dynkin criterion (1.3) because term-by-term integration of the power series expansion of the integrand yields $J=\infty$ if and only if $\sum_{j \geq 1} u_{j}(0) / j=\infty$, and this holds if and only if $\sum_{j \geq 1} U_{j}(0) / j^{2}=\infty$. Doney's criterion follows from Erickson's bound [11, Lemma 1] which in this case is $1 \leq M_{j} U_{j}(0) / j \leq 2$. The following result extends this by giving a criterion in terms of defining parameters of $\mathcal{X}$ which is equivalent to (3.11). 
THEOREM 3.9. Suppose that $1<m \leq \infty$ and $\{\lambda(j)\}$ is ultimately monotone. Then (3.11) holds if and only if

$$
K:=\sum_{j \geq 1} \frac{j}{M_{j}}\left(\frac{1}{\lambda(j)}-\frac{1}{\lambda(j+1)}\right)=\infty
$$

In addition, $\mathcal{X}$ is regular if and only if (3.13) holds.

PrOOF. We assume that $\lambda(j) \leq \lambda(j+1)$ for all $j \geq 1$, but alteration of some details in the sequel will show that this assumption entails no loss of generality. Abel's partial summation identity is

$$
\sum_{j=0}^{k} \frac{u_{j}(q)}{\lambda(j+1)}=\sum_{j=0}^{k} U_{j}(q)\left(\frac{1}{\lambda(j+1)}-\frac{1}{\lambda(j+2)}\right)+\frac{U_{k}(q)}{\lambda(k+2)} .
$$

Since $U_{j+1}(q) / U_{j}(q) \rightarrow 1$ as $j \rightarrow \infty$, we conclude that $K(q)<\infty$ if and only if

$$
K_{1}(q):=\sum_{j=0}^{\infty} U_{j}(q)\left(\frac{1}{\lambda(j)}-\frac{1}{\lambda(j+1)}\right)<\infty,
$$

provided $U_{k}(q) / \lambda(k)=O(1)$ as $k \rightarrow \infty$. Assume this for the moment and let $M_{j}(q)=\sum_{k=0}^{j} \sum_{l>k} w_{k}(q)$. Erickson's bound implies that $K_{1}(q)<\infty$ if and only if

$$
K_{2}(q)=\sum_{j \geq 1} \frac{j}{M_{j}(q)}\left(\frac{1}{\lambda(j)}-\frac{1}{\lambda(j+1)}\right)<\infty .
$$

It follows from (3.9) that $M_{j}(q)=\sum_{l \geq 1}\left(M_{l+j}-M_{l}\right) q^{l-1}$, and since $M_{l+j} / M_{j} \rightarrow 1$ as $j \rightarrow \infty$, it follows from the dominated convergence theorem that $M_{j}(q) / M_{j} \rightarrow$ $(1-q)^{-1}$. Thus we conclude that $K(q)<\infty$ if and only if $K<\infty$.

If $U_{k}(q) / \lambda(k) \rightarrow \infty$ through a subsequence, then $K(q)=\infty$. In addition,

$$
\sum_{j \geq k} U_{j}(q)\left(\frac{1}{\lambda(j)}-\frac{1}{\lambda(j+1)}\right) \geq U_{k}(q)\left(\frac{1}{\lambda(k)}-\frac{1}{\lambda(\infty)}\right) \rightarrow \infty
$$

so $K_{\mathrm{I}}(q)=\infty$ and hence $K=\infty$ too.

If $p_{0}=0$, then the assertion about regularity of $\mathcal{X}$ follows directly from Lemma $D$, Theorem 3.7 and from what has just been proved. If $K=\infty$ and $p_{0}>0$, then, using the notation in part (ii) of the proof of Theorem 3.3, let $\hat{p}_{0}=0$ and $\hat{p}_{j}=p_{j} / r_{0}$. Checking that $\hat{M}_{j}=\sum_{k=0}^{j} \sum_{l>k} \hat{p}_{k}=M_{j} / r_{0}$, we infer that the process $\mathcal{X}$ is regular, and (3.7) shows that $\mathcal{X}$ is regular too. The proof is complete. 
The criterion (3.13) is more general than the criterion that would follow directly from part (iii) of Doney's theorem because his proof of that result requires more assumptions about the jump rates. If $\lambda(j)=a j$ then we recover Doney's Corollary 2 for the MBP. Following Doney, if we assume that $1 / \lambda(j)=\int_{j}^{\infty} h(x) d x$, where $h(x)>0$ if $x \geq 1$, and also that

$$
0<\inf _{j \geq 1} \frac{h(j+x)}{h(j)} \leq \sup _{j \geq 1} \frac{h(j+x)}{h(j)}<\infty \quad(0 \leq x \leq 1),
$$

then $K<\infty$ if and only if $\sum_{j \geq 1} j h(j) / M_{j}<\infty$; compare with part (iii) of Doney's theorem. The above ratio bounds for $h$ replace Doney's condition $(*)$. If $\lambda(j+1)-$ $\lambda(j)=j^{\zeta-1} N(j)$ where $\zeta>0$ and $N(x)$ is slowly varying at infinity, then $K=\infty$ (and hence $\mathcal{X}$ is regular) if and only if $\sum_{j \geq 1}\left(\lambda(j) M_{j}\right)^{-1}=\infty$.

The criterion (3.13) directs attention to structural assumptions for the jump rates. Abel's lemma can be used to show that (3.13) holds if and only if

$$
K^{\prime}:=\sum_{j \geq 1} \frac{1}{\lambda(j)}\left(\frac{j+1}{M_{j+1}}-\frac{j}{M_{j}}\right)=\infty
$$

and the summands are eventually non-negative. This allows determination in terms of additional conditions on the jump distribution.

If $\mathcal{X}$ is irregular, then $\eta_{i} \rightarrow 0$ as $i \rightarrow \infty$, but finding the convergence rate is problematic, partly because it appears to depend in a detailed way on properties of the defining elements of $\mathcal{X}$. Consider the case where (2.8) holds and $J<\infty$; see (3.6). It is clear from (3.5) that $\eta_{i} \sim \int_{1-\epsilon}^{1}\left[s^{i} /(s-f(s)] \mu(d s)\right.$. It can be shown that the assumptions in Example 3.5 yield the estimates

$$
\eta_{i} \sim \begin{cases}\frac{\Gamma(\theta-\alpha)}{a i^{\theta-\alpha} L(i)} & \text { if } \theta>\alpha, \\ a^{-1} \int_{i}^{\infty} \frac{d z}{z L(z)} & \text { if } \theta=\alpha,\end{cases}
$$

where the integral is assumed finite if $\theta=\alpha$.

We have $\theta=1$ in the MBP case, and then $\left\{\eta_{i}\right\}$ is an appropriate norming constant for $\tau_{e}$ if $\alpha<1$, but not if $\alpha=1$. This assertion follows from Proposition 2 in Sagitov [28], as follows. Let $V(s)=\int_{s}^{1} d v /(v-f(v))$, which is finite since $\mathcal{Z}$ is irregular. The BK system takes the form $t \equiv V(F(s, t))-V(s)$. In particular, if $F(t):=F(1-, t)$, then $t \equiv V(F(t))$ and $P_{i}\left(\tau_{e} \leq t\right)=1-F^{i}(t)$. It follows that

$$
\lim _{i \rightarrow \infty} P_{i}\left(\tau_{e} \leq V(1-t / i)\right)=1-e^{-t} .
$$

This is the essential content of Sagitov's proposition. A further step yields the general limit result involving a nonlinear norming of the explosion time,

$$
\lim _{i \rightarrow \infty} P_{i}\left(i\left(1-F\left(\tau_{e}\right)\right) \leq t\right)=1-e^{-t} .
$$


It is straightforward to check that if $\alpha<1$, then $V(s) \sim(1-s)^{1-\alpha} /(1-\alpha) L\left((1-s)^{-1}\right)$ and hence (3.14) yields $\lim _{i \rightarrow \infty} P_{i}\left(\Gamma(2-\alpha) \tau_{e} / \eta_{i} \leq t\right)=1-\exp \left(-t^{1 /(1-\alpha)}\right)$. However, if $\alpha=1$, then $V(s) \sim a^{-1} \int_{1 /(1-s)}^{\infty} d z / z L(z)$, a slowly varying function of $(1-s)^{-1}$, and which does not permit a reduction similar to the case $\alpha<1$. However it can be shown that if $\alpha=1$ and $-\infty<v<\infty$, then

$$
\lim _{i \rightarrow \infty} P_{i}\left(\frac{L(i)}{\eta_{i}^{2}}\left(\tau_{e}^{-1}-\eta_{i}^{-1}\right) \leq v\right)=\exp \left(-e^{-v}\right)
$$

Some insight into the nonlinear case can be obtained if $p_{0}=0$, for then we have $\tau_{e}=\sum_{n \geq 0} \varepsilon_{n} / \lambda\left(i+S_{n}\right)$, where $S_{0}=0$ and the $\varepsilon_{n}$ are independent with the standard exponential law and indepdendent of the random walk $\left\{S_{n}\right\}$. We assume here that the series converges almost surely. If $\lambda(i)=R\left(e^{i}\right)$ where $R(x)=x^{\beta} N(x), \beta>0$, and $N(x)$ is slowly varying, then

$$
\lambda(i) \tau_{e} \stackrel{d}{\rightarrow} Z:=\sum_{n \geq 0} \varepsilon_{n} e^{-\beta S_{n}}<\infty \quad \text { (a.s.). }
$$

If $Z_{n}$ denotes the $n$-th partial sum of this random series then $Z_{n+1}=\varepsilon_{0}+e^{-\beta S_{1}} Z_{n}^{\prime}$ where $Z_{n}^{\prime} \stackrel{d}{=} Z_{n}$ and it is independent of $\left(\varepsilon_{0}, S_{1}\right)$. Hence $\left\{Z_{n}\right\}$ has the same one-dimensional laws as a random perpetuity. Similarly, we have $\lambda(i) \eta_{i} \rightarrow E(Z)=\left(1-e^{\beta} f\left(e^{-\beta}\right)\right)^{-1}$.

Next, suppose $1<m<\infty$ and $\lambda(i)=i^{\theta} M(i)$, where $\theta>1$ and $M(x)$ is slowly varying. Since $S_{n} / n \stackrel{\text { a.s. }}{\rightarrow} m-1$, it is fairly clear that

$$
\frac{1}{i} \sum_{n \geq 0} \frac{\lambda(i)}{\lambda\left(i+S_{n}\right)} \sim \frac{1}{i} \sum_{n \geq 0}\left(1+\frac{(m-1) n}{i}\right)^{-\theta} \rightarrow \int_{0}^{\infty}(1+(m-1) x)^{-\theta} d x,
$$

and hence that $\lambda(i) \eta_{i} / i \rightarrow[(\theta-1)(m-1)]^{-1}$. Considering $E_{i}\left[e^{-\zeta \lambda(i) \tau_{e} / i} \mid \mathcal{S}\right]$ in a similar manner shows that $\tau_{e} / \eta_{i} \stackrel{p}{\rightarrow} 1$.

\section{The stationary measure}

In this section we consider the existence of stationary measures $\left\{\mathcal{M}_{i}: i \geq 1\right\}$ for the transition semigroup $p_{i j}(t)=P_{i}\left(X_{t}=j\right)$, in other words, we seek non-trivial solutions $\mathcal{M}_{i} \geq 0$ of

$$
\mathcal{M}_{j}=\sum_{i \geq 1} \mathcal{M}_{i} p_{i j}(t) \quad(j \geq 1, t>0) .
$$

If $p_{0}=0$ and $j<i$, then $p_{i j}(t) \equiv 0$, so $\mathcal{M}_{j}=\sum_{i=1}^{j} \mathcal{M}_{i} p_{i j}(t) \rightarrow 0$ as $t \rightarrow \infty$. 
Hence we assume $p_{0}>0$ for the remainder of this section. Harris [15, page 111] showed that the MBP has a stationary measure $\left\{\sigma_{i}\right\}$ that is unique (up to a multiplicative constant) given by

$$
\sum_{i \geq 1} i \sigma_{i} s^{i-1}=\frac{p_{0} \sigma_{1}}{f(s)-s}, \quad(0 \leq s<q) .
$$

We set $p_{0} \sigma_{1}=1$. It follows that $\left\{\sigma_{i}\right\}$ is invariant for the q-matrix of $\mathcal{Z}$, equivalently, if $\mu_{i}:=i \sigma_{i}$ then $\left\{\mu_{i}\right\}$ is invariant for the jump chain $\mathcal{A}$,

$$
\sum_{i \geq 1} \mu_{i} p_{j-i+1}=\mu_{j} \quad(j \geq 1)
$$

If we define $\mathcal{M}_{i}=\mu_{i} / \lambda(i)$ for the general case, then (4.3) can be expressed in terms of the q-matrix $(1.1)$ as $\sum_{i \geq 1} \mathcal{M}_{i} q_{i j}=\sum_{i \geq 1} \mu_{i} p_{j-i+1}-\mu_{j} \equiv 0$, that is, $\left\{\mathcal{M}_{i}\right\}$ is invariant for $Q$.

We conjecture that $\left\{\mathcal{M}_{i}\right\}$ is invariant for the transition semigroup. This is equivalent to the following assertion. For some $v>0$, the system

$$
\sum_{i \geq 1} c_{i} q_{i j}=c_{j}, \quad(j \geq 1)
$$

and

$$
0 \leq c_{i} \leq \mathcal{M}_{i}
$$

has no non-trivial solution; see Anderson [1, page 195]. Our next result establishes this.

THEOREM 4.1. If $p_{0}>0$, then the measure $\left\{\mathcal{M}_{i}\right\}$ is the unique invariant measure for the transition semigroup of $\mathcal{X}$.

PROOF. The explicit form of (4.4) is

$$
\sum_{i=1}^{j+1} c_{i} \lambda(i) p_{j-i+1}=(\lambda(j)+\nu) c_{j}, \quad(j \geq 1)
$$

and since $p_{0}>0$, we can choose $c_{1}>0$ and solve recursively for $c_{j}(j \geq 2)$. Assume that (4.5) holds, equivalently, that $0 \leq d_{j} \leq \mu_{j}$ where $d_{j}:=\lambda(j) c_{j}$. Since $p_{0} \mu_{1}=1$, it follows from (4.2) that $\mu(s):=\sum_{j \geq 1} \mu_{j} s^{j}=s /(f(s)-s)$, and this is finite for $0 \leq s<q$. Thus $D(s):=\sum_{i \geq 1} d_{i} s^{i}<\infty$ if $s<q$, and hence (4.6) implies that $C(s):=\sum_{i \geq 1} c_{i} s^{i}<\infty$ and that $D(s)=[\alpha+\nu C(s)] \mu(s),(0 \leq s<q)$, where $\alpha:=p_{0} d_{1}$. Identifying coefficients of $s^{j}$ gives the identity

$$
d_{j}=\alpha \mu_{j}+v \sum_{i=1}^{j-1} c_{i} \mu_{j-i}, \quad(j \geq 2) .
$$


Consequently $d_{j}>\alpha \mu_{j}$, whence

$$
\frac{d_{j}}{\mu_{j}}>\alpha\left[1+\frac{\nu}{q^{j} \mu_{j}} \sum_{j=1}^{j-1} \frac{\mu_{i} q^{i}}{\lambda(i)} q^{j-i} \mu_{j-i}\right] .
$$

Yang [30] showed that if $m \neq 1$, then

$$
q^{j} \mu_{j} \uparrow(1-\beta)^{-1} \quad \text { where } \quad \beta=f^{\prime}(q)<1, \quad(j \uparrow \infty) .
$$

Consequently

$$
\liminf _{j \rightarrow \infty} \frac{d_{j}}{\mu_{j}} \geq \alpha\left[1+v \sum_{i \geq 1} \frac{\mu_{i} q^{i}}{\lambda(i)}\right],
$$

and we see from (4.7) that the series on the right-hand side converges if and only if $L=\sum_{i \geq 1} 1 / \lambda(i)<\infty$. So if $L=\infty$, then (4.5) is violated for all sufficiently large $j$. If $L<\infty$ then $v$ can be chosen so large that the right-hand side of (4.8) exceeds unity, and (4.5) again is violated.

If $m=1$, then $\mu(s)=s /(1-s)(1-w(s))$ where $w(s)=(1-f(s)) /(1-s)$ is a probability generating function. It follows that if $j \geq 1$, then $\mu_{j}=\sum_{k=0}^{j-1} u_{k}$ where $\left\{u_{j}\right\}$ is a (generalized) renewal sequence. Since $\mu(1)=\infty$, it follows that $\mu_{j+1} / \mu_{j} \rightarrow 1$ and Fatou's lemma can be used to obtain (4.8). The assertion then follows much as above. Thus $\left\{\mathcal{M}_{i}\right\}$ is invariant for the semigroup.

Conversely, any measure invariant for the semigroup is invariant for $Q$, and hence yields an invariant measure for the semigroup of $\mathcal{Z}$. However this measure is unique, thus completing the proof.

\section{FK uniqueness}

Assuming that $m>1$, Chen [5] showed the transition semigroup of $\mathcal{X}$ is FK-unique if (1.4) holds. The thrust of his proof can be considerably extended to demonstrate FK-uniqueness if $p_{0}>0$ and

$$
\sum_{j=1}^{\infty}\left(q^{j} \lambda(j)\right)^{-1}=\infty .
$$

We understand this identity as always holding if $p_{0}=0$. Chen et al. [8] proved the following result using a sequential criterion in Chen et al. [6, Theorem 3.5], and we now offer a different proof of their result.

THEOREM 5.1. The condition (5.1) is necessary and sufficient for $F K$-uniqueness. 
PROOF. We have only to prove that the system

$$
x_{j}=\sum_{i=0}^{j+1} x_{i} \lambda(i) p_{j-i+1}-\lambda(j) x_{j} \quad(j \geq 0),
$$

together with $x_{j} \geq 0$ and $\sum_{j=0}^{\infty} x_{j}<\infty$, has only the trivial solution if and only if (5.1) holds; see Anderson [1, page 82]. If $p_{0}=0$, then setting $j=1$ in (5.2) shows that $x_{0}=0$, and it follows by induction that $x_{j} \equiv 0$. The assertion is valid in this case.

Let $p_{0}>0$ and set $x_{0}=1$. It is clear then that (5.2) can be solved recursively for $x_{1}, x_{2}, \ldots$ Indeed, setting $C_{0}=0$ and $C_{j}=x_{j} \lambda(j)$ for $j \geq 1$, then (5.2) takes the recursive form

$$
p_{0} C_{j+1}=C_{j}+x_{j}-\sum_{i=1}^{j} C_{i} p_{j-i+1}
$$

where

$$
p_{0} C_{1}=1 \quad \text { and } \quad x_{1}=1 / p_{0} \lambda(1) \text {, }
$$

and so on. Define $\mu_{0}=0$ and recall that the invariant measure $\left\{\mu_{i}\right\}$ introduced in Section 4 is scaled so that $p_{0} \mu_{1}=1$. Now define numbers $x_{j}^{\prime}$ and $C_{j}^{\prime}=x_{j}^{\prime} \lambda(j)$ by $x_{0}^{\prime}=1$ and then, by recursion,

$$
C_{j}^{\prime}=\sum_{i=0}^{j-1} x_{i}^{\prime} \mu_{j-i} \quad(j \geq 1) .
$$

Some manipulation using (4.3) to evaluate the sum in (5.2) shows that (5.4) satisfies (5.2) and since $x_{0}=x_{0}^{\prime}$, it follows that $x_{j}^{\prime}=x_{j}$ and $C_{j}^{\prime}=C_{j}$ for all $j$. Thus (5.2) and (5.4) are equivalent, and we omit the primes. However (5.4) implies that $C_{j}>\mu_{j}$ and hence that $\sum_{j \geq 1} x_{j}>\sum_{j \geq 1} 1 / \mu_{j} \lambda(j)$. It follows from (4.7) that (5.1) implies that $\sum_{j \geq 1} x_{j}=\infty$ and hence the FK system has a unique solution.

Proving the converse requires three distinct steps. The first is a simple comparison result. Let $\bar{\lambda}(j)$ be jump rates satisfying $\bar{\lambda}(0)=0$ and $\lambda(j) \geq \bar{\lambda}(j)$ for all $j \geq 1$, and then let $\bar{x}_{0}=1$ and $\bar{x}_{j}$ and $\bar{C}_{j}$ be constructed from the analogous version of (5.4). Then (5.3) implies that $C_{1}=\bar{C}_{1}$ and $x_{1}=\bar{x}_{1} \bar{\lambda}(1) / \lambda(1) \leq \bar{x}_{1}$. It follows from (5.4) that if $x_{i} \leq \bar{x}_{i}(i=0, \ldots, j-1)$, then $C_{j} \leq \bar{C}_{j}$, whence

$$
x_{j} \leq \frac{\bar{C}_{j}}{\lambda(j)}=\frac{\bar{x}_{j} \bar{\lambda}(j)}{\lambda(j)} \leq \bar{x}_{j}
$$

Hence, by induction, these inequalities hold for all $j$ and, in particular,

$$
X(s):=\sum_{j=0}^{\infty} x_{j} s^{j} \leq \bar{X}(s):=\sum_{j=0}^{\infty} \bar{x}_{j} s^{j} .
$$


Second, it follows from (4.7) that $\mu(s):=\sum_{j \geq 1} \mu_{j} s^{j}$ converges if $s<q$, and together with the ratio test we infer that the function

$$
\bar{X}(s):=1+\sum_{j=1}^{\infty} A^{j} \prod_{l=1}^{j} \mu\left(q^{l} s\right)
$$

converges if $s<1$ and that it has a power series representation as above with coefficients $\bar{x}_{j} \geq 0$. Some manipulation shows that $\bar{X}(s)$ solves a functional equation which can be expressed as

$$
A^{-1}(\bar{X}(s / q)-1)=\mu(s) \bar{X}(s), \quad(s<q) .
$$

Identifying coefficients of $s^{j}$ yields $\left(A q^{j}\right)^{-1} \bar{x}_{j}=\sum_{i=0}^{j-1} \bar{x}_{i} \mu_{j-i}$, so we conclude that (5.4) is satisfied with $\bar{\lambda}(j):=\left(A q^{j}\right)^{-1}$.

Finally, assume that the series (5.1) converges and denote its sum by $A^{-1}$. Then $\lambda(j) \geq \bar{\lambda}(j)$ and we conclude that $X(s)<\infty$ if $s<1$. Writing (5.4) as

$$
q^{j} C_{j}=\sum_{i=0}^{j-1} x_{i} q^{i} q^{j-i} \mu_{j-i},
$$

it follows from the monotone convergence theorem and (4.7) that

$$
q^{j} C_{j} \rightarrow L:=\frac{X(q)}{1-\beta}<\infty
$$

that is, $x_{j} \sim L / q^{j} \lambda(j)$ and hence that $\sum_{j \geq 0} x_{j}<\infty$, thus completing the proof.

\section{Resurrection}

Let $\mathcal{Y}=\left(Y_{t}\right)$ be the process obtained by restarting or resurrecting independent copies of $\mathcal{X}$ whenever they hit $\{0\}$. Specifically, let $\rho=\lambda(0)>0$ and $\left\{h_{j}: j \geq 1\right\}$ be a probability mass function. Then $\mathcal{Y}$ is the minimal process for the q-matrix $\tilde{Q}$ where $\tilde{q}_{i j}=q_{i j}$ as given by (1.1) for $i, j \geq 1, \tilde{q}_{00}=-\rho$, and $\tilde{q}_{0 j}=\rho h_{j}$ if $j \geq 1$. Thus $Y_{t}=X_{t}$ if $t<\tau_{0}$, and if $\tau_{0}<\tau_{e}$, then $Y_{t}=0$ if $\tau_{0} \leq t<\tau_{0}+R_{1}$, where $R_{1}$ is independent of $\left(Y_{t}: t \leq \tau_{0}\right)$ and it has an exponential distribution with mean $\rho^{-1}$, $P\left(Y_{\tau_{0}+R_{1}}=j\right)=h_{j}$, and the process evolves further in a similar way. See Chen [4] for the case (1.4), and Pakes and Tavaré [26, Section 4] for the general notion of return processes.

A sample path of $\mathcal{Y}$ has a finite first infinity $\tau_{\infty}$ if and only if one of the $\mathcal{X}$ excursions explodes, and hence $\mathcal{Y}$ is regular if and only if $\mathcal{X}$ is regular. 
THEOREM 6.1. The resurrected process $\mathcal{Y}$ is regular if $m \leq 1$. If $1<m \leq \infty$ and (2.8) holds, then $\mathcal{Y}$ is regular if and only if $J=\infty$ (see (3.6)); if $1<m<\infty$, then $\mathcal{Y}$ is regular if and only if (3.8) holds; and if $m=\infty$ and $\{\lambda(j): j \geq 1\}$ is ultimately monotone, then $\mathcal{Y}$ is regular if and only if (3.13) holds.

The case (2.8) subsumes Proposition 1.1 of Chen [4]. It is clear that if the conditions for explosion are satisfied, then $\xi_{i}:=E_{i}\left(\tau_{\infty}\right)<\infty$ for all $i$. The next result gives an integral representation for this quantity for the case (2.8). Let $h(s)=\sum_{j \geq 1} h_{j} s^{j}$.

THEOREM 6.2. Let $1<m \leq \infty$ and (2.8) and (3.6) both hold. Then

$$
\xi_{i}=\rho^{-1} G_{i 0}+\int_{0}^{1} \frac{s^{i}-(1-h(s)) G_{i 0}}{s-f(s)} \mu(d s)
$$

where

$$
G_{i 0}=\frac{q^{i}}{1-h(q)}
$$

Proof. Let $\mathcal{W}=\left\{W_{n}\right\}$ denote the jump chain of $\mathcal{Y}$ (that is, $\mathcal{W}$ is a resurrected version of $\mathcal{A})$, let $E_{i}\left(s^{W_{n}}\right)=R_{i}^{(n)}(s)=\sum_{j \geq 0} r_{i j}^{(n)} s^{j}$ and $G_{i j}=\sum_{n \geq 0} r_{i j}^{(n)}$. Clearly

$$
\xi_{i}=\sum_{j \geq 0} \frac{G_{i j}}{\lambda(j)}=\frac{G_{i 0}}{\rho}+\int_{0}^{1}\left(R_{i}(s, 1)-G_{i 0}\right) \mu(d s)
$$

where $R_{i}(s, x)=\sum_{n \geq 0} R_{i}^{(n)}(s) x^{n}$. Observe that

$$
R_{i}^{(n+1)}(s)=E_{i}\left(s^{W_{n}} ; W_{n}>0\right) b(s)+r_{i 0}^{(n)} h(s)=R_{i}^{(n)}(s) b(s)-r_{i 0}^{(n)}(b(s)-h(s)),
$$

and hence that

$$
R_{i}(s, x)=\frac{s^{i}-x R_{i}(0, x)(b(s)-h(s))}{1-x b(s)} .
$$

Matching zeros as in the proof of Theorem 2.1 yields

$$
R_{i}(0, x)=\frac{g^{i}(x)}{1-x h(g(x))}
$$

and (6.2) follows by setting $x=1$. Similarly

$$
R_{i}(s, 1-)=\frac{s^{i}-G_{i 0}(b(s)-h(s))}{1-b(s)},
$$

and (6.1) follows. 
It is clear from the sample path behaviour of $\mathcal{Y}$ that the non-negative integers comprise a communicating class of states, and that the states are recurrent if $m \leq 1$ and they are transient if $m>1$. In the former case it follows from (2.8) and (2.9) that the mean recurrence time of $\{0\}$ is

$$
m_{00}=\rho^{-1}+\sum_{i \geq 1} h_{i} H_{i}=\rho^{-1}+\int_{0}^{1} \frac{1-h(s)}{f(s)-s} \mu(d s) .
$$

THEOREM 6.3. Suppose that (2.8) holds. Then $\mathcal{Y}$ is recurrent if and only if $m \leq 1$, in which case it is positive recurrent if and only if $m_{00}<\infty$. If this holds, then the generating function of the limiting-stationary law is

$$
\Pi(s)=\pi_{0}\left(1+\rho s \int_{0}^{1} \frac{1-h(s y)}{f(s y)-s y} \mu(d y)\right)
$$

where

$$
\pi_{0}=\left[1+\rho \int_{0}^{1} \frac{1-h(y)}{f(y)-y} \mu(d y)\right]^{-1} .
$$

PROOF. We need only demonstrate (6.4). If $m_{00}<\infty$, then the limiting-stationary law $\left\{\pi_{j}: j \geq 0\right\}$ solves $\sum_{i \neq j} \pi_{i} \tilde{q}_{i j}=\lambda(j) \pi_{j}$. Multiplying by $s^{j}$ and summing over $j \geq 0$ gives the identity

$$
\sum_{i \geq 1} \pi_{i} \lambda(i) s^{i-1}=\pi_{0} \rho \frac{1-h(s)}{f(s)-s} .
$$

Replace $s$ with $s y$ and integrate both sides with respect to $\mu(d y)$ over $[0,1]$. This cancels the $\lambda(i)$ factors on the left-hand side, giving $\left(\Pi(s)-\pi_{0}\right) / s$, and (6.4) and (6.5) now follow.

If $\mu(d s)=\zeta(s) d s$, then

$$
\left.\Pi(s)=\pi_{0}\left(1+\rho s \int_{0}^{s} \frac{1-h(v)}{f(v)-v} \zeta(v / s) d v\right)\right) .
$$

This expression together with Theorem 6.3 extends the principal results in Sections 3 and 4 of Chen [4]. Indeed we can say rather more.

Let $\left\{\bar{\pi}_{j}\right\}$ denote the stationary distribution for the resurrected MBP, that is, where $\lambda(i)=a i$ if $i \geq 1$, and similarly for $\bar{q}_{i j}$ and related quantities. Taking $\zeta(s) \equiv a^{-1}$ in (6.7) we see that $\bar{\Pi}^{\prime}(s) / \bar{\pi}_{0}=(\rho / a)(1-h(s)) /(f(s)-s)$, and reference to (6.4) and (2.8) yields the identification $\lambda(j) \pi_{j} / \pi_{0}=a j \bar{\pi}_{j} / \bar{\pi}_{0},(j \geq 1)$. Observing that $\left\{\bar{\pi}_{j} / \bar{\pi}_{0}\right\}$ is a stationary measure for $\bar{Q}$, then using this identity to define $\pi_{j} / \pi_{0}$ for any positive set of jump rates $\lambda(j),(j \geq 1)$, it is easily checked that the invariance equations $\sum_{i \geq 0} \pi_{i} \tilde{q}_{i j}=0$ are satisfied for all $j \geq 0$. We thus get the following result. 
THEOREM 6.4. If $m \leq 1$, then $\left\{\bar{\sigma}_{j}: j \geq 0\right\}$, defined by.

$$
\sum_{j \geq 1} \bar{\sigma}_{j} s^{j}=1+\frac{\rho s}{a} \int_{0}^{s} \frac{1-h(v)}{f(v)-v} d v
$$

is the unique stationary measure for $\overline{\mathcal{Y}}$. If $S:=\sum_{j \geq 1} j \bar{\sigma}_{j} / \lambda(j)<\infty$, then $\mathcal{Y}$ is positive recurrent with limiting-stationary law given by $\pi_{0}=(1+a S)^{-1}$ and $\pi_{j}=\pi_{0} a j \bar{\sigma}_{j} / \lambda(j),(j \geq 1)$.

Suppose $m_{00}<\infty$. Then $\mathcal{Y}$ (specifically, its transition semigroup) is strongly ergodic if $\sup _{i \geq 0} \sum_{j \geq 0}\left|p_{i j}(t)-\pi_{j}\right| \stackrel{t \rightarrow \infty}{\longrightarrow} 0$.

This occurs if and only if $\sup _{i \geq 0} H_{i}<\infty$ (Anderson [1, page 215]). Thus the next result follows from Theorems $2.2-2.5$.

THEOREM 6.5. If $m \leq 1$ and $m_{00}<\infty$, then $\mathcal{Y}$ is strongly ergodic if and only if case (a) of Theorems 2.2 or 2.3 hold. If (2.8) also holds then $\mathcal{Y}$ is strongly ergodic if and only if the limit (2.11) is finite.

EXAMPLE 6.6. Suppose (2.8) holds with $\mu(d s)=(1-s)^{\theta-1} L\left((1-s)^{-1}\right)$ and that $1-h(s)=(1-s)^{\delta} M\left((1-s)^{-1}\right)$, where $\delta>0,0 \leq \theta \leq 1$, and $L(x)$ and $M(x)$ are slowly varying at infinity. If $m<1$, then $m_{00}<\infty$ if and only if

$$
\int_{1}^{\infty} x^{-\delta-\theta} L(x) M(x) d x<\infty
$$

and hence $\mathcal{Y}$ is ergodic if $\delta+\theta>1$, not ergodic if $\delta+\theta<1$, and if $\delta+\theta=1$ then it is ergodic if and only if $\int_{1}^{\infty} x^{-1} L(x) M(x) d x<\infty$. If it is ergodic, then it is strongly ergodic if $\theta>1$, and not so if $\theta \leq 1$. If $m=1$ and $\gamma>0$, then all the above assertions hold provided the critical value 1 is replaced by 2 .

\section{Acknowledgment}

I thank Anyue Chen for allowing me access to his unpublished work during the preparation of this paper, and for a useful remark on the penultimate version.

\section{References}

[1] W. J. Anderson, Continuous-time Markov chains (Springer-Verlag, New York, 1991).

[2] L. Breiman, 'On transient Markov chains with application to the uniqueness problem for Markov processes', Ann. Math. Statist. 28 (1957), 499-503. 
[3] N. G. de Bruijn and P. Erdös, 'On a recursion formula and on some Taubèrian theorems', $J$. Research. Nat. Bur. Standards 50 (1953), 161-164.

[4] A. Chen, 'Ergodicity and stability of generalised Markov branching processes with resurrection', J. Appl. Prob. 39 (2002), 786-803.

[5] - 'Uniqueness and extinction properties of generalized Markov branching processes', $J$. Math. Anal. Appl. 274 (2002), 482-494.

[6] A. Chen, J. Li and N. I. Ramesh. 'General Harris regularity criterion for non-linear Markov branching processes', Statist. Probab. Letters 76 (2005), 446-552.

[7] __ 'Uniqueness and extinction of weighted Markov branching processes', Methodol. Comput. Appl. Prob. 7 (2005), 489-516.

[8] A. Chen, P. Pollett, J. Li and H. Zhang, 'A remark on the uniqueness of weighted Markov branching processes', J. Appl. Prob. 44 (2007), 274-283.

[9] R.-R. Chen, 'An extended class of time-continuous branching processes', J. Appl. Prob. 34 (1997), 14-23.

[10] R. A. Doney, 'A note on some results of Schuh', J. Appl. Prob. 21 (1984), 192-196.

[11] K. B. Erickson, 'The strong law of large numbers when the mean is undefined', Trans. Amer. Math. Soc. 185 (1973), 371-381.

[12] A. M. Etheridge, An introduction to superprocesses (American Mathematical Society, Providence, RI, 2000).

[13] A. M. Garsia. S. Orey and E. Rodemich, 'Asymptotic behaviour of successive coefficients of some power series', Illinois J. Math. 6 (1962), 620-629.

[14] K. Hamza and F. C. Klebaner, 'Conditions for integrability of Markov chains', J. Appl. Probab. 32 (1995), 541-547.

[15] T. E. Harris. The theory of branching processes (Springer-Verlag, Berlin, 1963).

[16] N. Ikeda, M. Nagasawa and S. Watanabe, 'Branching Markov processes II', J. Math. Kyoto Univ. 8 (1968), 365-410.

[17] G. Kersting and F. C. Klebaner, 'Sharp conditions for non explosions and explosions in Markov jump processes', Ann. Prob. 23 (1995), 268-272.

[18] P. Küster, 'Generalized Markov branching processes with state-dependent offspring distributions', Z. Wahrsch. Verw: Gebiete 64 (1983), 475-503.

[19] N. Lenz, 'Extinction probability, regularity and asymptotic growth of Markovian populations', J. Appl. Prob. 18 (1981), 1-18.

[20] C. Lipow, 'Limiting diffusions for population-size dependent branching processes', J. Appl. Prob. 14 (1977), 14-24.

[21] A. G. Pakes, 'Conditional limit theorems for a left-continuous random walk', J. Appl. Prob. 10 (1973), 39-53.

[22] _ _ 'The branching-catastrophe process'. Stoch. Processes. Appl. 23 (1986), 1-33.

[23] — 'Asymptotic results for the extinction time of Markov branching processes allowing emigration, I. Random walk decrements', Adv. Appl. Prob. 21 (1989), 243-269.

[24] _ - 'Divergence rates for explosive birth processes', Stoch. Processes Appl. 41 (1992), 91-99.

[25] _ ' Explosive Markov branching processes: Entrance laws and limiting behaviour', Adv. Appl. Prob. 25 (1993), 737-756.

[26] A. G. Pakes and S. Tavaré, 'Comments on the age distribution of Markov processes'. Adv. Appl. Prob. 13 (1981), 681-703.

[27] I. Reinhard, 'The qualitative behaviour of some slowly growing population-dependent Markov branching processes', in: Stochastic Modelling in Biology (ed. P. Tautu) (World Scientific, Singapore, 1990) pp. 267-277.

[28] S. M. Sagitov, 'On an explosive branching process', Theor. Prob. Appl. 40 (1996), 575-577. 
[29] T. H. Savits, 'The explosion problem for branching Markov processes', Osaka J. Math. 6 (1969), 375-395.

[30] Y. N. Yang, 'Asymptotic properties of the stationary measure of a Markov branching process', $J$. Appl. Prob. 10 (1973), 447-450.

[31] V. M. Zolotarev, 'More exact statements of several theorems in the theory of branching processes', Theor. Prob. Appl. 2 (1957), 245-253.

School of Mathematics and Statistics

University of Western Australia

35 Stirling Highway

Crawley WA 6009

Australia

e-mail: pakes@maths.uwa.edu.au 